\title{
Aplicação da audiometria troncoencefálica na detecção de perdas auditivas retrococleares em trabalhadores de manutenção hospitalar expostos a ruído
}

\author{
Auditory brainstem responses evaluation of retrocochlear disease \\ in workers from a general hospital exposed to noise
}

Victor Luiz da Silveira ${ }^{1}$

Volney de Magalhães Câmara ${ }^{1}$

Cláudia Maria Valete Rosalino ${ }^{2}$

${ }^{1}$ Universidade Federal do Rio de Janeiro. Av.

Brigadeiro Trompowsky s/ $\mathrm{n}^{\circ}$, Praça da Prefeitura

Universitária, Ilha do

Fundão. 21949-900 Rio de Janeiro RJ.

victorluiz.silveira@gmail.com

${ }^{2}$ Instituto de Pesquisas

Clínicas da Fundação

Oswaldo Cruz.

\begin{abstract}
The main purpose of this cross-seccional study was to investigate the presence of retrocochlear disease in a group of maintenance workers from a general hospital, who presents a history of noise exposure. Thirty one workers of three engineering sections with age range from 25 to 60 years and continuous noise exposure from 2 and 45 years, were examined. The evaluation included an audiometric occupational selection and auditory brainstem responses (ABR). ABR abnormalities were detected in seven patients $(22.6 \%)$ and it was found latency increase of waves III (14.3\%) and V (28.6\%), and interpeak prolongation I-III (71.4\%), III-V (28.6\%) and I-V (85.7\%). Among 35 ears with normal audition - right, left or both -, four $(11,4 \%)$ ears presented retrocochlear disease. The high retrocochlear disease prevalence in workers exposed to noise lead us to suppose that this disturbance is more frequent than usually found; therefore it is underestimated in workers diagnostic evaluation. The presence of this kind of disturbance, even with the absence of audiometric alterations, suggests that $A B R$ is more sensitive than the tonal audiometry for noise induced hearing loss investigation.
\end{abstract}

Key words Noise, Auditory brainstem responses, Noise-induced hearing loss, Retrocochlear disease, Deafness, Worker health
Resumo O objetivo principal deste estudo seccional foi averiguar a presença de comprometimento auditivo retrococlear num grupo de trabalhadores de manutenção de um hospital de grande porte com histórico de exposição a ruído. Foram avaliados 31 trabalhadores de três setores da divisão de engenharia entre 25 e 60 anos e com exposição contínua a ruído entre 2 e 45 anos. $O$ processo avaliativo contou com triagem audiométrica ocupacional e audiometria troncoencefálica (ATE). Foram detectadas anormalidades na ATE em sete pacientes $(22,6 \%)$, caracterizadas por aumento de latências de ondas III (14,3\%) e V (28,6\%); prolongamento dos interpicos I-III (71,4\%), III$V(28,6 \%)$ e $I-V(85,7 \%)$. Das 35 orelhas com audição normal (três unilateralmente e 32 bilateralmente), quatro $(11,4 \%)$ apresentaram comprometimento retrococlear. A alta prevalência de comprometimento retrococlear no grupo induz supor que tal distúrbio seja mais frequente que o encontrado e esteja sendo subestimado na investigação diagnóstica desses trabalhadores. A ocorrência desses resultados sem a presença de alterações audiométricas sugere que a ATE seja mais sensivel que a audiometria tonal na investigação de perda auditiva provocada por ruído, por isso sua utilização deva ser incentivada.

Palavras-chave Ruído, Audiometria troncoencefálica (ATE), Perda auditiva provocada por ruido (PAPR), Doenças retrococleares, Surdez, Saúde do trabalhador 


\section{Introdução}

A perda auditiva provocada por ruído (PAPR) é a doença ocupacional mais comum e evitável apesar de irreversível - em todo o mundo. A exposição ao ruído e seus efeitos sobre a saúde são considerados na atualidade um problema de saúde pública de importância crescente ${ }^{1}$.

As modificações no processo de trabalho que vêm ocorrendo mundialmente dificultam o controle da saúde e da segurança dos trabalhadores em seus ambientes de trabalho. Dentre tais modificações, destacam-se aquelas relacionadas ao campo tecnológico e as alterações nas relações de trabalho ${ }^{2}$. A relação entre o ruído produzido pela maquinaria e a perda auditiva adquirida pelos trabalhadores, muitas vezes em concomitância com outros efeitos na saúde, como por exemplo estresse, irritabilidade, hipertensão arterial, desordens psicofisiológicas e de interação social ${ }^{3,4}$, é um provável fator potencializador do aumento da taxa de morbidade entre o operariado.

A perda auditiva provocada por ruído consiste numa perda auditiva sensorioneural bilateral, geralmente simétrica, resultante de ruído contínuo ou interrompido, que atinge inicialmente as frequências de 3.000 e $6.000 \mathrm{~Hz}$, podendo ocasionar perdas de até $40 \mathrm{dBNA}$ (decibel nível de audição) nas frequências baixas e 75 dBNA nas frequências altas ${ }^{5}$. A PAPR pode ser definida como "uma patologia cumulativa e insidiosa, que cresce ao longo de anos de exposição associada comumente ao ambiente de trabalho", causada por qualquer exposição ao longo de oito horas por dia, que exerça uma média de 90 dBNPS (decibel nível de pressão sonora), regularmente, por um período de vários anos, resultando alterações mecânicas ou metabólicas ${ }^{6}$.

A audiometria troncoencefálica (ATE) tem se mostrado útil no diagnóstico diferencial da perda auditiva sensorioneural, fornecendo informações que podem objetivamente indicar se a lesão é situada em nível coclear ou retrococlear ${ }^{7}$. Através da ATE, foi observado em trabalhadores expostos a ruído ausência de ondas precoces (I e III $)^{8}$, aumento das latências das ondas I, III e V9, especialmente da onda V10, e aumento dos intervalos interpicos I-III, III-V e I-V ${ }^{9,10}$.

Neste sentido, e levando-se em consideração as funções da audição na proteção do indivíduo e na comunicação social, entre outras, este estudo tem como objetivo principal avaliar a perda auditiva provocada por ruído em um grupo ocupacional formado por trabalhadores de manutenção de um hospital universitário de grande porte, bem como a contribuição da ATE para os serviços de saúde na identificação e avaliação da localização da lesão deste tipo de perda auditiva nesse grupo de trabalhadores.

\section{Métodos}

Foi realizado um estudo epidemiológico descritivo seccional ou de prevalência. Consistiu em verificar as alterações neurais decorrentes da exposição continuada ao ruído em trabalhadores de manutenção de um hospital universitário do município do Rio de Janeiro (RJ), levando-se em consideração variáveis selecionadas como jornada de trabalho e uso de equipamento de proteção individual.

A população estudada inicialmente era composta por 38 trabalhadores do sexo masculino da divisão de engenharia do hospital, sendo 28 diaristas e dez plantonistas distribuídos da seguinte forma: dez funcionários da carpintaria, nove funcionários das caldeiras e 19 funcionários da refrigeração. Entretanto, dois trabalhadores da carpintaria afastaram-se do trabalho por motivos diversos, três do setor de caldeiras recusaram-se a participar do estudo e dois da refrigeração tiveram seus contratos de trabalho vencidos no período da coleta dos dados. Dos 31 trabalhadores avaliados (oito do grupo carpintaria, seis do grupo caldeiras e 17 do grupo refrigeração), 23 atuavam como diaristas em jornadas de até oito horas, e oito atuavam em plantões de 24 por 96 horas. A carpintaria é composta por trabalhadores com idade entre 34 e 60 anos e exposição a ruído entre 2 e 38 anos; as caldeiras por trabalhadores de 41 a 59 anos de idade e de 6 a 29 de exposição; e a refrigeração por trabalhadores entre 25 e 59 anos de idade e de 7 a 45 anos de exposição.

Foram levantados os riscos à saúde dos funcionários e descritos os locais de trabalho: a oficina de carpintaria, a praça de caldeiras e a oficina de refrigeração. Ainda que se tenha observado integralmente tais ambientes e também se tenha arrolado todas as possíveis situações de risco físico, químico, mecânico e ergonômico, foi dada maior ênfase à descrição dos níveis de ruído (risco físico). Entretanto, é importante mencionar que os trabalhadores das caldeiras e da carpintaria, apesar do ruído do ambiente, também estão expostos a substâncias como o pó de serra, cola e solventes - carpintaria - e óleo BPF - caldeiras. Apenas os trabalhadores da carpintaria sofrem risco potencial de ototoxicidade, devido ao contato com os solventes. 
As medições dos níveis de ruído dos tipos contínuo e intermitente foram feitas com decibelímetro Lutran SL 4001 calibrado a $94 \mathrm{~dB}(\mathrm{~A})$. Os trabalhadores foram submetidos às seguintes fases do processo avaliativo: (1) preenchimento do questionário autoaplicativo, composto de questões referentes à anamnese otológica e ocupacional; (2) otoscopia realizada por médico otorrinolaringologista, que teve por objetivo principal a inspeção meticulosa do meato acústico externo para a exclusão de rolha ceruminosa ou corpo estranho; (3) triagem audiométrica por via aérea realizada por fonoaudiólogo; e (4) ATE, para a determinação do topodiagnóstico de lesões na via auditiva, realizada por ambos. Seguiu-se a recomendação de Melnick ${ }^{11}$, que aponta que regulamentos internacionais da atualidade prescrevem o repouso mínimo de 14 horas sem exposição de ruído do local de trabalho para que se estabeleça o audiograma de base. Desta forma, todas as fases do processo avaliativo foram realizadas pela manhã, antes de os trabalhadores se dirigirem ao seu trabalho.

De acordo com as recomendações da Portaria $\mathrm{n}^{\circ}$ 19, de 9 de abril de 1998 (Ministério do Trabalho) ${ }^{12}$, referente à NR 7 (Programa de Controle Médico de Saúde Ocupacional), o exame audiométrico foi realizado pela via aérea nas frequências de 500, 1.000, 2.000, 3.000, 4.000, 6.000 e 8.000 $\mathrm{Hz}$, e por via óssea nas frequências de 500, 1.000, 2.000, 3.000 e $4.000 \mathrm{~Hz}$, caso seja detectada alguma alteração no exame por via aérea. Entretanto, por se tratar de uma triagem audiométrica precedente à realização da ATE, apenas com função de determinar quais indivíduos eram normais e quais tinham queda no limiar de audibilidade, não foi avaliada a via óssea, não obstante a presença de alterações verificadas no limiar tonal aéreo. Para as avaliações, foram utilizados um audiômetro de um canal Beltone 112 com fones Beltone e uma sala silenciosa, porém sem isolamento acústico.

Como a pesquisa objetivava verificar a presença de comprometimento retrococlear em trabalhadores com histórico ocupacional de exposição a ruído, não foram investigados comprometimentos condutivos. Tendo em vista que a triagem tonal por via área visava somente enquadrar os indivíduos nas categorias audição normal e audição alterada, não se classificaram as alterações nos limiares auditivos como condutivas, sensorioneurais ou mistas, e sim apenas como quedas de limiares aéreos do tipo: leve (25 a 40 dBNA), moderada (45 a 70 dBNA), severa (75 a 90 dBNA), profunda (95 dBNA em diante) e combinações (leve a moderada e leve a severa).
A fim de detectar se as perdas auditivas observadas eram retrococleares, lançou-se mão da ATE, exame eletrofisiológico que capta potenciais bioelétricos no nervo auditivo e tronco encefálico evocados por estímulo auditivo (clique), permitindo assim identificar disfunções neurais nessas localizações. A ATE foi realizada no aparelho Amplaid MK-15, com fones TDH 49, em sala sem isolamento acústico. Os participantes foram acomodados em decúbito dorsal, em posição confortável na maca com a cabeça e o pescoço bem apoiados e da forma mais relaxada possível, com o intuito de diminuir ao máximo a atividade muscular a fim de se evitar interferência de respostas. Os locais de fixação dos eletrodos foram limpos com solução de éter-álcool a 50\%, seguindo-se o uso de esponja de aço para leve escarificação da pele. Em seguida aplicou-se pasta de betonita nos eletrodos de superfície; eles foram fixados na pele limpa com fita adesiva ${ }^{13}$. O estímulo foi apresentado na velocidade de 11 cliques/segundo, visto que o objetivo era determinar o topodiagnóstico, e para isso é necessário maior resolução e fidedignidade na apresentação das ondas. Foram obtidos os valores de latência absoluta das ondas I, III e V, os valores das latências interpicos I-III, III-V e $\mathrm{I}-\mathrm{V}$, e a diferença interaural I-V.

Os dados coletados através do questionário autoaplicativo, da triagem audiométrica por via aérea e do exame audiométrico eletrofisiológico foram armazenados em banco de dados, processados e analisados através do programa computacional EPI-Info versão 6.04, referenciado pelo Center for Disease Control and Prevention (CDC). Foram obtidos valores de média, mediana e moda dos ambientes avaliados, assim como as médias das idades dos trabalhadores de cada setor e do tempo de exposição, e a razão de prevalência, o Qui-quadrado e o valor-p de algumas variáveis em relação à alteração da ATE.

O presente estudo foi aprovado pelo Comitê de Ética em Pesquisa do Núcleo de Estudos de Saúde Coletiva da Universidade Federal do Rio de Janeiro, contemplando as recomendações da Resolução nº 196/96 do Ministério da Saúde.

\section{Resultados}

O ruído ambiental médio mensurado na carpintaria sem o funcionamento das máquinas foi de 63.25 decibéis avaliado com filtro na posição $\mathrm{A}$ $(\mathrm{dB}(\mathrm{A}))$. Os valores encontrados em diferentes pontos da oficina foram 52, 64, 65 e $72 \mathrm{~dB}(\mathrm{~A})$ $($ mediana $=64.50 \mathrm{~dB}(\mathrm{~A}))$. Em relação ao ruído 
mensurado próximo à maquinaria, na serra circular de bancada com esquadra encontrou-se na faixa entre 94 e $100 \mathrm{~dB}(\mathrm{~A})$ (média e mediana $=97$ $\mathrm{dB}(\mathrm{A})$ ) e na serra circular de bancada sem esquadra foi de aproximadamente $93 \mathrm{~dB}(\mathrm{~A})$.

Quanto à praça de caldeiras, visto que o operador não permanece por muito tempo próximo à caldeira, ficando enclausurado numa saleta, foi mensurado o nível de ruído dentro desta, com a caldeira ligada correspondendo a $80 \mathrm{~dB}(\mathrm{~A})$, e desligada correspondendo a $60 \mathrm{~dB}(\mathrm{~A})$. O ruído da caldeira medido próximo ao painel de controle foi de $91 \mathrm{~dB}(\mathrm{~A})$. Os valores medidos em diferentes pontos deste local foram $81,81,83,83,86$ e 87 $\mathrm{dB}(\mathrm{A})$ (mediana $=83 \mathrm{~dB}(\mathrm{~A})$ e $\operatorname{moda}=81$ e 83 $\mathrm{dB}(\mathrm{A})$ ). O ruído ambiental médio medido na praça de caldeiras foi de $83.50 \mathrm{~dB}(\mathrm{~A})$, e na oficina de caldeiras, que também serve de sala de repouso, foi de $73.50 \mathrm{~dB}(\mathrm{~A})$. Os valores medidos em diferentes pontos da oficina de caldeiras e sala de repouso foram 70, 71, 76 e $77 \mathrm{~dB}(\mathrm{~A})$ (mediana = $73.50 \mathrm{~dB}(\mathrm{~A}))$.

No tocante à refrigeração, o ruído ambiental médio mensurado numa das casas de máquinas (a) foi de $85.66 \mathrm{~dB}(\mathrm{~A})$ e na outra (b) foi de 78.66 $\mathrm{dB}(\mathrm{A})$, enquanto na oficina foi de $64 \mathrm{~dB}(\mathrm{~A})$. Os valores medidos em diferentes pontos da casa de máquinas (a) foram 85, 85 e $87 \mathrm{~dB}(\mathrm{~A})$ (mediana $=86 \mathrm{~dB}(\mathrm{~A})$ e moda $=85 \mathrm{~dB}(\mathrm{~A}))$. Os valores medidos em diferentes pontos da casa de máquinas (b) foram 77, 79 e $80 \mathrm{~dB}(\mathrm{~A})$ (mediana $=78.66$ $\mathrm{dB}(\mathrm{A}))$. Os valores medidos em diferentes pontos na oficina foram 63, 63 e $65 \mathrm{~dB}(\mathrm{~A})$ (mediana $=64 \mathrm{~dB}(\mathrm{~A})$ e moda $=63 \mathrm{~dB}(\mathrm{~A}))$.

Vale ressaltar que o risco químico a que os trabalhadores da carpintaria estão expostos tem implicação audiológica, o que justifica a necessidade de considerá-los um grupo à parte, já que existe a concomitância de exposição ao ruído e a solvente.

As médias de idade encontradas na carpintaria, nas caldeiras e na refrigeração foram, respectivamente, 48,8, 49,0 e 44,3 anos. As médias do tempo de exposição observada nos respectivos setores foram 17,7, 22,3 e 20,2 anos.

Não houve entre os grupos algum indivíduo que portasse um laudo otorrinolaringológico ou médico ocupacional contendo o diagnóstico de PAPR. Dos 31 trabalhadores avaliados, 7 (22,6\%) apresentaram alteração retrococlear à ATE. No grupo de indivíduos com anormalidades na ATE, havia aqueles com audição normal e ainda aqueles com quedas de limiares aéreos que variavam de leve a moderada, uni ou bilateral. Das 31 orelhas com audição normal, quatro $(11,4 \%)$ apresentaram comprometimento retrococlear.

Após a remoção de cerume em alguns trabalhadores, não foram verificadas alterações à otoscopia. As doenças comuns da infância (DCI) caxumba, sarampo e catapora - foram confirmadas em 24 pacientes $(77,4 \%)$, sendo que destes cinco $(20,8 \%)$ estavam entre aqueles sete que apresentaram alteração retrococlear. Como o número de pacientes com história de DCI e lesão retrococlear confirmada é muito pequeno, não é possível ratificar significância estatística entre as variáveis DCI e ATE. Quanto ao uso de medicamentos pelos trabalhadores no último ano, nenhum deles se enquadrava no grupo de medicamentos ototóxicos, sendo que houve dois relatos de uso de analgésico, dois de anti-inflamatórios, dois de anti-hipertensivos, um de antibióticos, um de insulina, um de medicação anticerume e um de antivertiginoso, não sendo estatisticamente relevantes tais dados. Não foram relevadas as exposições pregressas a medicamentos para os fins deste estudo.

As tabelas 1 e 3 sintetizam a descrição dos demais resultados obtidos na anamnese e nas avaliações. As tabelas 2 e 4 correspondem àqueles, porém, referentes aos trabalhadores do grupo da carpintaria. Para proteger a identidade dos participantes, estes foram identificados nas tabelas pelo número do questionário. A Tabela 5 relaciona algumas variáveis relevantes aos resultados obtidos através da avaliação eletrofisiológica.

\section{Discussão e conclusão}

A partir desses resultados, pudemos constatar que, dos 15 trabalhadores que apresentaram diminuição no limiar tonal aéreo de audibilidade, 33,3\% mostraram alguma alteração neural observada pela ATE. No entanto, como se pode observar nas tabelas 1 a 5, a distribuição dos dados obtidos foi heterogênea, o que significa que não foi possível estabelecer inferências estatísticas. Além disso, o número de pessoas estudadas não permitiria tais inferências. Não houve, portanto, valores estatisticamente significantes, os quais pudessem comprovar quaisquer hipóteses de associação de informações semiológicas, patológicas ou mesmo de histórico de exposição ao ruído, à detecção de alterações retrococleares. 
Tabela 1. Síntese dos resultados obtidos na anamnese: refrigeração e caldeiras (exposição somente a ruído) Rio de Janeiro, 2005.

\begin{tabular}{|c|c|c|c|c|c|c|}
\hline $\mathbf{N}^{\circ}$ & Idade & $\begin{array}{l}\text { Setor } \\
\text { (grupo) }\end{array}$ & Jornada & $\begin{array}{c}\text { Exposição a } \\
\text { ruído (em anos) }\end{array}$ & Sintomas otológicos & Outras situações \\
\hline 1 & 49 & $\mathrm{R}$ & $8 \mathrm{~h}$ & 27 & OD: otalgia & - \\
\hline 2 & 43 & $\mathrm{R}$ & $8 \mathrm{~h}$ & 12 & OD e OE: secreção & MLR / FLR \\
\hline 3 & 48 & $\mathrm{R}$ & $24 / 96 h$ & 11 & - & - \\
\hline 4 & 40 & $\mathrm{R}$ & $6 \mathrm{~h}$ & 25 & - & MLR / FLR / TLR \\
\hline 5 & 39 & $\mathrm{R}$ & $24 / 96 h$ & 11 & - & FLR \\
\hline 6 & 50 & $\mathrm{R}$ & $24 / 96 \mathrm{~h}$ & 29 & Tontura & FLR / TLR \\
\hline 7 & 50 & $\mathrm{R}$ & $6 \mathrm{~h}$ & 30 & - & - \\
\hline 8 & 38 & $\mathrm{R}$ & $8 \mathrm{~h}$ & 11 & - & FLR \\
\hline 9 & 36 & $\mathrm{R}$ & $24 / 96 \mathrm{~h}$ & 12 & OE: pressão & FLR \\
\hline 10 & 25 & $\mathrm{R}$ & $8 \mathrm{~h}$ & 7 & - & FLR \\
\hline 11 & 44 & $\mathrm{R}$ & $8 \mathrm{~h}$ & 13 & - & TLR \\
\hline 12 & 49 & $\mathrm{R}$ & $8 \mathrm{~h}$ & 16 & OE: hipoacusia & MLR \\
\hline 13 & 57 & $\mathrm{R}$ & $8 \mathrm{~h}$ & 35 & - & $\begin{array}{l}\text { Walkman } \\
\text { FLR / TLR }\end{array}$ \\
\hline 14 & 47 & $\mathrm{R}$ & $8 \mathrm{~h}$ & 21 & $\begin{array}{l}\text { OD: hipoacusia } \\
\text { OE: hipoacusia e secreção }\end{array}$ & MLR / FLR / TLR \\
\hline 15 & 32 & $\mathrm{R}$ & $12 \mathrm{~h}$ & 10 & - & - \\
\hline 16 & 59 & $\mathrm{R}$ & $8 \mathrm{~h}$ & 45 & OD e OE: secreção & FLR \\
\hline 17 & 52 & $\mathrm{R}$ & $8 \mathrm{~h}$ & 34 & - & MLR / FLR \\
\hline 18 & 41 & CAL & $8 \mathrm{~h}$ & 16 & - & FLR \\
\hline 19 & 41 & CAL & 24/96h & 29 & $\begin{array}{l}\text { OD: hipoacusia, zumbido e } \\
\text { pressão } \\
\text { OE: hipoacusia e zumbido. } \\
\text { Tontura }\end{array}$ & FLR \\
\hline 20 & 59 & CAL & $8 \mathrm{~h}$ & 6 & $\begin{array}{l}\text { OD: zumbido } \\
\text { OE: zumbido e perfuração }\end{array}$ & FLR \\
\hline 21 & 58 & CAL & 24/96h & 29 & OD e OE: hipoacusia & FLR \\
\hline 22 & 47 & CAL & $10 \mathrm{~h}$ & 27 & $\begin{array}{l}\text { OD: hipoacusia } \\
\text { OE: hipoacusia e pressão }\end{array}$ & - \\
\hline 23 & 48 & CAL & 24/96h & 27 & $\begin{array}{l}\text { OD: hipoacusia, otalgia, } \\
\text { zumbido, otorragia. } \\
\text { Tontura }\end{array}$ & $\begin{array}{l}\text { Usa protetor } \\
\text { auricular }\end{array}$ \\
\hline
\end{tabular}

Obs.: $\mathrm{N}^{\circ}$ - identificação do paciente pelo número do questionário; R - setor de refrigeração; CAL - setor de caldeiras; OD orelha direita; OE - orelha esquerda; FLR - frequenta local ruidoso; MLR - mora em local ruidoso; NL - normalidade; TLR trabalha em outro local ruidoso; DLI - diferença interaural I-V.

Tabela 2. Síntese dos resultados obtidos na anamnese: carpintaria (exposição a ruído e a produto químico) Rio de Janeiro, 2005.

\begin{tabular}{cccccll}
\hline $\mathbf{N}^{\circ}$ & Idade & $\begin{array}{c}\text { Setor } \\
\text { (grupo) }\end{array}$ & Jornada & $\begin{array}{c}\text { Exposição } \\
\text { (em anos) }\end{array}$ & \multicolumn{1}{c}{ Sintomas otológicos } & Outras situações \\
\hline 24 & 60 & $\mathrm{C}$ & $8 \mathrm{~h}$ & 14 & OD e OE: hipoacusia e zumbido & MLR \\
25 & 40 & $\mathrm{C}$ & $24 / 96 \mathrm{~h}$ & 15 & - & FLR \\
26 & 51 & $\mathrm{C}$ & $7 \mathrm{~h}$ & 30 & OD: otalgia, zumbido e pressão & FLR \\
27 & 34 & $\mathrm{C}$ & $8 \mathrm{~h}$ & 6 & OE: zumbido & FLR \\
28 & 47 & $\mathrm{C}$ & $8 \mathrm{~h}$ & 28 & OE: otalgia & MLR \\
29 & 52 & $\mathrm{C}$ & $8 \mathrm{~h}$ & 2 & - & Usa protetor auricular \\
30 & 47 & $\mathrm{C}$ & $8 \mathrm{~h}$ & 6 & - & MLR / FLR / TLR \\
31 & 51 & $\mathrm{C}$ & $8 \mathrm{~h}$ & 38 & - & - \\
\hline
\end{tabular}

Obs.: $\mathrm{N}^{\circ}$ - identificação do paciente pelo número do questionário; $\mathrm{R}$ - setor de refrigeração; CAL - setor de caldeiras; OD orelha direita; OE - orelha esquerda; FLR - frequenta local ruidoso; MLR - mora em local ruidoso; NL - normalidade; TLR trabalha em outro local ruidoso; DLI - diferença interaural I-V. 
Tabela 3. Síntese dos resultados obtidos nas avaliações auditivas: refrigeração e caldeiras (exposição somente a ruído) - Rio de Janeiro, 2005.

\begin{tabular}{|c|c|c|c|c|}
\hline $\mathbf{N}^{\circ}$ & Idade & Setor (grupo) & Triagem audiométrica & ATE \\
\hline \multirow[t]{2}{*}{1} & 49 & $\mathrm{R}$ & OD: NL & OD: aumento de III, V e I-V \\
\hline & & & OE: queda leve & OE: aumento de III, V, I-III e I-V \\
\hline 2 & 43 & $\mathrm{R}$ & OD e OE: NL & OD e OE: NL \\
\hline 3 & 48 & $\mathrm{R}$ & OD e OE: NL & OD e OE: NL \\
\hline 4 & 40 & $\mathrm{R}$ & OD e OE: NL & OD e OE: NL \\
\hline 5 & 39 & $\mathrm{R}$ & OD e OE: NL & OD e OE: NL \\
\hline \multirow[t]{2}{*}{6} & 50 & $\mathrm{R}$ & OD: queda leve & OD e OE: NL \\
\hline & & & OE: queda leve a moderada & \\
\hline \multirow[t]{2}{*}{7} & 50 & $\mathrm{R}$ & OD: queda leve & OD e OE: NL \\
\hline & & & OE: queda leve & \\
\hline 8 & 38 & $\mathrm{R}$ & OD e OE: NL & OD e OE: NL \\
\hline \multirow[t]{2}{*}{9} & 36 & $\mathrm{R}$ & OD: queda moderada & OD e OE: NL \\
\hline & & & OE: queda moderada & \\
\hline 10 & 25 & $\mathrm{R}$ & OD e OE: NL & OD e OE: NL \\
\hline 11 & 44 & $\mathrm{R}$ & OD e OE: NL & $\begin{array}{l}\text { OD: aumento de I-V e da DLI I-V } \\
\text { OE: NL }\end{array}$ \\
\hline \multirow[t]{2}{*}{12} & 49 & $\mathrm{R}$ & OD: NL & OE: aumento de I-V \\
\hline & & & OE: queda leve a moderada & OD: NL \\
\hline 13 & 57 & $\mathrm{R}$ & OD e OE: NL & OD e OE: NL \\
\hline \multirow[t]{2}{*}{14} & 47 & $\mathrm{R}$ & OD: queda leve a moderada & OD e OE: NL \\
\hline & & & OE: queda moderada & \\
\hline 15 & 32 & $\mathrm{R}$ & OD e OE: NL & OD e OE: NL \\
\hline \multirow[t]{2}{*}{16} & 59 & $\mathrm{R}$ & OD: queda leve a moderada & OD e OE: NL \\
\hline & & & OE: queda leve & \\
\hline 17 & 52 & $\mathrm{R}$ & OD e OE: NL & OD e OE: NL \\
\hline 18 & 41 & CAL & OD e OE: NL & OD e OE: NL \\
\hline \multirow[t]{2}{*}{19} & 41 & CAL & OD: queda leve a moderada & OD e OE: NL \\
\hline & & & OE: queda leve a moderada & \\
\hline \multirow[t]{2}{*}{20} & 59 & CAL & OD: queda leve & OD: aumento de III-V e I-V \\
\hline & & & OE: queda leve & OE: aumento de V, III-V e I-V \\
\hline \multirow[t]{2}{*}{21} & 58 & CAL & OD: queda moderada & OD e OE: NL \\
\hline & & & OE: queda leve a moderada & \\
\hline \multirow[t]{2}{*}{22} & 47 & CAL & OD e OE: NL & OD: aumento de I-V \\
\hline & & & & OE: aumento de I-III e I-V \\
\hline \multirow[t]{2}{*}{23} & 48 & CAL & OD: queda leve a moderada & OD e OE: NL \\
\hline & & & OE: queda leve a moderada & \\
\hline
\end{tabular}

Obs.: $\mathrm{N}^{\circ}$ - identificação do paciente pelo número do questionário; $\mathrm{R}$ - setor de refrigeração; $\mathrm{CAL}$ - setor de caldeiras; $\mathrm{OD}$ orelha direita; OE - orelha esquerda; FLR - frequenta local ruidoso; MLR - mora em local ruidoso; NL - normalidade; TLR trabalha em outro local ruidoso; DLI - diferença interaural I-V.

Considerando que o Ministério da Saúde, através das recomendações da Portaria no 3.751 , de 23 de novembro de $1990^{14}$, presentes no anexo I da NR 15 - atividades e operações insalubres -, prevê um limite máximo de exposição durante oito horas diárias de $85 \mathrm{~dB}$, apenas os trabalhadores que atuam em uma das casas de máquinas da refrigeração estariam expostos potencialmente a um ruído lesivo para o sistema auditivo, visto que nesse local encontrou-se um ruído ambiental médio próximo ao limite estabelecido. $\mathrm{O}$ mes- mo não ocorre com os trabalhadores da carpintaria, já que o tempo de operação da maquinaria é breve, não obstante as altas médias de ruído obtidas com o funcionamento dos aparelhos. Apesar de ser obrigatório o uso do protetor auricular, este era ignorado pelos três grupos de trabalhadores, sendo que apenas dois trabalhadores de setores distintos o utilizavam, não tendo sido observada associação significativa entre o uso do protetor auricular e a ausência de alteração retrococlear. 
Tabela 4. Síntese dos resultados obtidos nas avaliações auditivas: carpintaria (exposição a ruído e a produto químico) - Rio de Janeiro, 2005.

\begin{tabular}{|c|c|c|c|c|}
\hline $\mathbf{N}^{\circ}$ & Idade & Setor (grupo) & Triagem audiométrica & ATE \\
\hline \multirow[t]{2}{*}{24} & 60 & $\mathrm{C}$ & OD: queda leve a moderada & OD: aumento de I-V \\
\hline & & & OE: queda leve a severa & OE: aumento de I-III e I-V \\
\hline 25 & 40 & $\mathrm{C}$ & OD e OE: NL & OD e OE: NL \\
\hline 26 & 51 & $\mathrm{C}$ & OD: queda moderada & OD e OE: NL \\
\hline 27 & 34 & $\mathrm{C}$ & $\begin{array}{l}\text { OE: queda leve } \\
\text { OD: queda leve a moderada } \\
\text { OE: NL }\end{array}$ & OD e OE: NL \\
\hline 28 & 47 & $\mathrm{C}$ & OD e OE: NL & OD e OE: NL \\
\hline 29 & 52 & $\mathrm{C}$ & OD e OE: NL & OD e OE: NL \\
\hline 30 & 47 & $\mathrm{C}$ & $\begin{array}{l}\text { OD: queda leve a moderada } \\
\text { OE: queda leve a moderada }\end{array}$ & OD e OE: aumento de I-III, III-V e I-V \\
\hline 31 & 51 & $\mathrm{C}$ & OD e OE: NL & OD e OE: NL \\
\hline
\end{tabular}

Obs.: $\mathrm{N}^{\circ}$ - identificação do paciente pelo número do questionário; $\mathrm{R}$ - setor de refrigeração; $\mathrm{CAL}$ - setor de caldeiras; OD orelha direita; OE - orelha esquerda; FLR - frequenta local ruidoso; MLR - mora em local ruidoso; NL - normalidade; TLR trabalha em outro local ruidoso; DLI - diferença interaural I-V.

Tabela 5. Distribuição dos resultados dos testes de audiometria eletrofisiológica (ATE) segundo variáveis selecionadas - Rio de Janeiro, 2005.

\begin{tabular}{|c|c|c|c|c|c|c|c|c|c|}
\hline & \multicolumn{2}{|c|}{ Normalidade } & \multicolumn{2}{|c|}{ Alteração retrococlear } & \multicolumn{2}{|c|}{ Total } & \multicolumn{3}{|c|}{ Valores estatísticos } \\
\hline & $\mathrm{N}^{\mathrm{O}}$ & $\%$ & $\mathrm{~N}^{\mathrm{O}}$ & $\%$ & $\mathrm{~N}^{\mathrm{O}}$ & $\%$ & $\mathrm{RP}$ & Valor -p & $\chi^{2}$ \\
\hline População estudada & - & - & - & - & - & - & - & 0,44 & 2,71 \\
\hline 25 a 35 anos & 3 & 12,5 & 0 & 0,0 & 3 & 9,7 & - & - & - \\
\hline 36 a 45 anos & 8 & 33,3 & 1 & 14,3 & 9 & 29,0 & - & - & - \\
\hline 46 a 55 anos & 10 & 41,7 & 4 & 57,1 & 14 & 42,2 & - & - & - \\
\hline Acima de 55 anos & 3 & 12,5 & 2 & 28,6 & 5 & 16,1 & - & - & - \\
\hline Tempo de exposição & - & - & - & - & - & - & - & 0,27 & 3,91 \\
\hline 2 a 12 anos & 9 & 37,5 & 2 & 28,6 & 11 & 35,5 & - & - & - \\
\hline 13 a 22 anos & 3 & 12,5 & 3 & 42,9 & 6 & 19,4 & - & - & - \\
\hline 23 a 32 anos & 8 & 33,3 & 2 & 28,6 & 10 & 32,3 & - & - & - \\
\hline Acima de 32 anos & 4 & 16,7 & 0 & 0,0 & 4 & 12,9 & - & - & - \\
\hline Triagem audiométrica & - & - & - & - & - & - & 2,04 & 0,16 & 1,92 \\
\hline Normal & 14 & 58,3 & 2 & 28,6 & 16 & 51,6 & - & - & - \\
\hline Alterada & 10 & 41,7 & 5 & 71,4 & 15 & 48,4 & - & - & - \\
\hline DCI & - & - & - & - & - & - & 1,11 & 0,67 & 0,19 \\
\hline Confirmadas & 19 & 79,2 & 5 & 71,4 & 24 & 77,4 & - & - & - \\
\hline Negadas & 5 & 20,8 & 2 & 28,6 & 7 & 22,6 & - & - & - \\
\hline Traumatismo cefálico & - & - & - & - & - & - & 1,75 & 0,55 & 0,36 \\
\hline Confirmado & 6 & 25,0 & 1 & 14,7 & 7 & 22,6 & - & - & - \\
\hline Negado & 18 & 75,0 & 6 & 85,7 & 24 & 77,4 & - & - & - \\
\hline Zumbido & - & - & - & - & - & - & 0,58 & 0,48 & 0,49 \\
\hline Confirmado & 4 & 16,7 & 2 & 28,6 & 6 & 19,4 & - & - & - \\
\hline Negado & 20 & 83,3 & 5 & 71,4 & 25 & 80,6 & - & - & - \\
\hline Tontura & - & - & - & - & - & - & - & 0,32 & 0,97 \\
\hline Confirmada & 3 & 12,5 & 0 & 0,0 & 3 & 9,7 & - & - & - \\
\hline Negada & 21 & 87,5 & 7 & 100 & 28 & 90,3 & - & - & - \\
\hline Exposição a ruído fora do HU & J - & - & - & - & - & - & 1,05 & 0,85 & 0,04 \\
\hline Confirmada & 18 & 75,0 & 5 & 71,4 & 23 & 74,2 & - & - & - \\
\hline Negada & 6 & 25,0 & 2 & 28,6 & 8 & 25,8 & - & - & - \\
\hline Uso de protetor auricular & - & - & - & - & - & - & - & 0,43 & 0,62 \\
\hline Confirmado & 2 & 8,3 & 0 & 0,0 & 2 & 6,5 & - & - & - \\
\hline Negado & 22 & 91,7 & 7 & 100 & 29 & 93,5 & - & - & - \\
\hline
\end{tabular}

Obs.: $\mathrm{N}^{\mathrm{O}}$ - número; DCI - doenças comuns da infância; RP - razão de prevalência; $\chi^{2}$ - Teste Qui-quadrado. 
O local de trabalho exerceu pouca influência nos resultados. Outros fatores, como exposição ao ruído fora do ambiente de trabalho e o histórico de DCI, foram descartados por estarem presentes em grande parte dos avaliados que não possuíam ATE alterada, não sendo, portanto, possível fazer inferências estatísticas com essas variáveis.

A literatura especializada é exaustiva no tocante à relação entre PAPR e as alterações morfofisiopatológicas da cóclea, especificamente do órgão sensorial de Corti. Utilizando a ATE, muitas pesquisas avaliaram cobaias expostas a ruído através da observação do limiar eletrofisiológico $^{15-18}$. Todavia, poucos autores destacaram-se na análise de distúrbios das vias auditivas centrais, as quais conduzem a informação auditiva da cóclea ao córtex auditivo primário, sobretudo em grupos de trabalhadores com exposição a ruído ocupacional ${ }^{7-9,19}$. Nessa perspectiva de avaliação auditiva retrococlear de um grupo específico de trabalhadores de hospital expostos a ruído ocupacional através das ondas da ATE, é possível que este trabalho seja o pioneiro em língua portuguesa.

Os trabalhadores que tiveram alterações na ATE, correspondendo a 38,7\% das orelhas avaliadas, pertenciam aos três setores, estando concentrados numa faixa etária que vai de 44 a 60 anos (média $=46,2$ anos). Seu tempo de exposição estende-se de 6 a 27 anos (média $=20,1$ anos). Tais resultados não são compatíveis com a pesquisa realizada por Noorhassim et al. ${ }^{8}$, na qual foram encontradas alterações nas ondas da ATE em $72 \%$ das orelhas avaliadas em um grupo de 22 trabalhadores. Esta discrepância provavelmente está relacionada ao fato de que todos esses indivíduos que eles analisaram já tinham PAPR diagnosticada, diferente do presente estudo, em que $51,6 \%$ dos trabalhadores investigados tinham audição normal bilateralmente. Além disso, apesar de o tempo médio de exposição a ruído encontrado pelos autores (média e desviopadrão $=21+12,7$ anos) ser semelhante ao nosso, a média de idade dos trabalhadores que eles investigaram (média e desvio-padrão $=62,1+$ 9,9 anos) era maior que a que encontramos, o que pode ter contribuído para esse alto percentual de orelhas afetadas, já que também podem ter sofrido influência de alterações decorrentes de presbiacusia.

Dentre os sete exames de ATE considerados anormais, verificou-se que não houve aumento de latência de onda I; apenas um (14,3\%) apresentou aumento de onda III, dois $(28,6 \%)$ apre- sentaram aumento de onda $V$, cinco $(71,4 \%)$ apresentaram aumento do intervalo interpico I-III, dois $(28,6 \%)$ apresentaram aumento do interpico III$\mathrm{V}$, seis $(85,7 \%)$ apresentaram aumento do interpico I-V e um (14,3\%) apresentou aumento significativo de diferença interaural I-V. Não evidenciamos ausência de ondas em nenhum dos trabalhadores investigados. No estudo de Noorhassim et $a l .{ }^{8}$ foram observados aumentos da latência da onda III $(46,9 \%)$, do intervalo III-V $(37,5 \%)$, do intervalo I-V $(31,2 \%)$, da latência absoluta da onda I $(25 \%)$ e da onda V (25\%) e a ausência de ondas I $(25 \%)$ e III $(9,4 \%)$. Como é possível observar pela comparação dos resultados, ambos os estudos tiveram alta frequência de aumento de interpico I-V, que é indicativo de degeneração de ambas as vias auditivas periféricas e centrais. É importante destacar que, no entanto, nosso estudo mostrou maior frequência de alterações no intervalo I-III, o que é indicativo de lesão do tronco encefálico ${ }^{20}$, até aproximadamente o complexo olivar superior, enquanto o estudo deles observou maior frequência de alterações no intervalo III-V, o que é indicativo de lesão do tronco encefálico a partir do complexo olivar superior, até aproximadamente o colículo inferior. Em outras palavras, encontramos alterações mais periféricas, enquanto Noorhassim et al. ${ }^{8}$ encontraram alterações mais centrais. O motivo desta discrepância deve ser o mesmo anteriormente exposto.

Da mesma forma, Xu et al. ${ }^{7}$ encontraram 9,1\% de aumento de latência de onda V no grupo de trabalhadores expostos ao ruído por eles investigado, que era composto por 22 trabalhadores expostos ao ruído com PAPR diagnosticada, com idade média de 51 anos e tempo médio de exposição de 26 anos, assim como encontraram ausência de onda I em sujeitos com perdas auditivas de leve a moderada entre 1 e $4 \mathrm{kHz}$. Mais uma vez, as médias de idade e de exposição menores encontradas em nosso estudo, associadas ao fato de que nossos trabalhadores não tinham PAPR comprovada, alguns até apresentando audiometria tonal normal, podem justificar não termos observado ausência de ondas. Vale ressaltar, no entanto, que apesar disto observamos um número maior de alterações nos padrões de normalidade de latências absolutas e interpicos em relação a Xu et al. ${ }^{7}$, fato este sem explicação plausível.

Attias e Pratt ${ }^{9}$ pesquisaram a ATE antes e depois de exposição a ruído ocupacional por 9 a 14 meses, em 31 indivíduos de 18 a 21 anos com audição inicialmente normal. Seus resultados mostraram prolongamento da latência absoluta da onda I e dos intervalos interpicos I-III e III-V 
após este período de exposição. Estes achados estão compatíveis com os nossos, com exceção do aumento de latência de onda I. É relevante destacar que as maiores ocorrências de distúrbios na ATE do presente estudo podem ser decorrentes das maiores médias de faixa etária e de tempo de exposição, além do que a maioria dos trabalhadores investigados por Attias e Pratt ${ }^{9}$ usava protetores auriculares, bem diferente do que ocorria com a nossa amostra.

Almadori et al. ${ }^{19}$ estudaram 54 trabalhadores (108 orelhas) diagnosticados com PAPR entre 25 e 51 anos e com tempo de exposição a ruído mínimo de cinco anos, submetendo-os à audiometria tonal liminar e logoaudiometria, à imitanciometria e à ATE com estímulo de 21 cliques/segundo, a fim de averiguar a possibilidade de envolvimento retrococlear. Os dados da ATE indicaram que 12 orelhas $(11,1 \%)$ apresentaram ausência de componentes precoces, sendo dez de onda I e dois de ondas I e III, e que cinco orelhas $(4,6 \%)$ apresentaram ausência total de potenciais evocados. Além disso, realizaram estudos radiológicos e neurológicos nos trabalhadores, o que permitiu excluir comprometimento retrococlear nos que tinham ausência de ondas. Tais resultados não são compatíveis com o presente estudo, pois não foi verificada a ausência de ondas em nenhum dos trabalhadores. Vale ressaltar que nossas avaliações foram executadas com um estímulo lento (11 cliques/segundo), o que permite melhor individualização das ondas em relação ao estímulo mais rápido por eles utilizados, o que pode ter favorecido a ausência de ondas do estudo deles. Além disso, a comparação entre os estudos ficou prejudicada, uma vez que eles não definiram através de médias a faixa etária e o tempo de exposição.

O único estudo brasileiro que fomos capazes de encontrar na literatura ${ }^{21}$ evidenciou alteração da morfologia das ondas I, III e V, respectivamente em $13,75 \%, 12,5 \%$ e $12,5 \%$, e aumento de intervalo I-V em $15 \%$ das oitenta orelhas analisadas de 42 pacientes, com mediana de 49 anos, referenciados a uma clínica otorrinolaringológica para investigação de PAPR, sem tempo de exposição definido. Nosso estudo não foi capaz de observar nenhuma alteração de morfologia das ondas e observou aumento de intervalo interpico I-V em $9,7 \%$ das 62 orelhas analisadas. Destacamos que a nossa mediana foi de 47 anos e que encontramos níveis audiométricos normais em $56,4 \%$ das 62 orelhas pesquisadas, enquanto no estudo de Ferreira et al. ${ }^{21}$ apenas $8,7 \%$ das orelhas analisadas apresentavam níveis audiométricos normais, o que justifica a menor frequência por nós encontrada de aumento de intervalo I-V.

Não é possível, através dos dados apresentados e da sua análise, afirmar que haja uma relação efetiva entre a exposição a ruído e os distúrbios retrococleares no grupo estudado, apesar de já ter sido descrito na literatura internacional que a exposição continuada pode danificar não apenas as células ciliadas externas cocleares, mas também as vias auditivas periféricas e centrais. Para determinar se os casos retrococleares são oriundos da exposição, seria necessário submeter os indivíduos a exames complementares para se realizar diagnóstico por exclusão. Dentre os exames, pode-se citar alguns laboratoriais para excluir, por exemplo, etiologia metabólica, e exames de imagem como a tomografia computadorizada e a imagem de ressonância magnética, a fim de descartar lesões tumorais ou outras alterações histológicas como etiologias do distúrbio.

A alta prevalência de comprometimento retrococlear em trabalhadores expostos a ruído nos faz supor que tal distúrbio seja mais frequente que o encontrado e esteja sendo, portanto, subestimado na investigação diagnóstica desses trabalhadores. A presença do distúrbio no grupo, mesmo com ausência de alterações de limiares auditivos, sugere que a ATE seja mais sensível que a audiometria tonal na investigação de PAPR e que por isso sua utilização deva ser incentivada. Novos estudos nesta área serão capazes de verificar a veracidade desta hipótese.

Determinar a prevalência de alterações retrococleares e ratificá-las como uma das manifestações da PAPR é de fundamental importância para o desenvolvimento de programas de conservação auditiva, dada a irreversibilidade das perdas auditivas resultantes de lesões no nervo auditivo e nas vias auditivas do tronco cerebral. 


\section{Colaboradores}

VL Silveira, VM Câmara e CMV Rosalino participaram igualmente de todas as etapas da elaboração do artigo.

\section{Referências}

1. World Health Organization (WHO). [site da Internet]. 2004. [acessado 2007 jul 8]. Disponível em: http://www.who.int/mediacentre/factsheets/fs258/en/

2. Câmara VM, Tambellini AT, Castro HÁ, Waissman W. Saúde ambiental e saúde do trabalhador: epidemiologia das relações entre a produção, o ambiente e a saúde. In: Rouquayrol MZ, organizador. Epidemiologia \& saúde. $6^{a}$ ed. Rio de Janeiro: Medsi; 2003. p. 469-497.

3. Santana VS, Barberino JL. Exposição ocupacional ao ruído e hipertensão arterial. Rev Saude Publica 1995; 29(6):478-487.

4. Corrêa Filho HR, Costa LS, Hoehne EL, Pérez MAG, Nascimento LCR, Moura EC. Perda auditiva induzida por ruído e hipertensão em condutores de ônibus. Rev Saude Publica 2002; 36(6):693-701.

5. Silva AA, Costa EA. Avaliação da surdez ocupacional. Rev Ass Med Brasil 1998; 44(1):65-68.

6. Oliveira JAA. Fisiologia clínica da audição: cóclea ativa. In: Nudelmann AA, Costa EA, Seligman J, Raul NI, organizadores. PAIR: perda auditiva induzida pelo ruído. Porto Alegre: Bagagem Comunicação; 1997. p. 101-142.

7. Xu ZM, Vinck B, de Vel E, van Cauwenberge P. Mechanisms in noise-induced permanent hearing loss: an evoked otoacoustic emission and auditory brainstem response study. J Laringol Otol 1998; 112(12):1154-1161.

8. Noorhassim I, Kaga K, Nishimura K. Pure-tone audiometry and auditory brainstem responses in noise-induced deafness. American Journal of Otolaringology 1996; 17(1):31-35.

9. Attias J, Pratt H. Auditory evoked potentials and audiological follow up of subjects developing noiseinduced permanent threshold shift. Audiology 1984; 23:498-508.

10. Janczewski G, Kochaneck K, Dawidowicz J, Tanziarello A, Dobrzynski P, Bardadin J. Examinaton of reaction time: auditory brainstem responses ABR in noise induced temporary and permanent threshold shift. Med Pr 1994; 42(1):37-42.

11. Melnick W. Saúde auditiva do trabalhador. In: Katz J. Tratado de audiologia clínica. $4^{\text {a }}$ ed. São Paulo: Manole; 1999. p. 529-547.

12. Brasil. Ministério do Trabalho. Secretaria de Segurança e Saúde no Trabalho. Portaria ${ }^{\circ} 19$, de 9 de abril de 1998. Diretrizes e parâmetros mínimos para avaliação e acompanhamento da audição em trabalhadores expostos a niveis de pressão sonora elevados. NR 7: Programa de Controle Médico de Saúde Ocupacional. Brasília: Ministério da Saúde; 1998. (Anexo 1, Quadro II).
13. Valete CM. Audiometria de tronco encefálico com tone pip: uma estimativa do limiar auditivo eletrofisiológico em $1.000 \mathrm{~Hz}$ [dissertação]. Rio de Janeiro: UFRJ/ FM; 1999.

14. Brasil. Portaria $n^{\circ} 3.751$, de 23 de novembro de 1990. Limites de tolerância para ruído contínuo ou intermitente. NR 15: atividades e operações insalubres. Brasília: Ministério da Saúde; 1990. (Anexo 1).

15. Boettcher FA. Auditory brainstem response correlates of resistence to noise-induced hearing loss in Mongolian gerbils. J Accoust Soc Am 1993; 94(6):3207-3214.

16. Ngan EM, May BJ. Relationship between the auditory brainstem response and auditory nerve thresholds in cats with hearing loss. Hear Res 2001; 156(12):44-52.

17. Fraenkel R, Freeman S, Sohmer H. Use of ABR threshold and OAEs in detection of noise induced hearing loss. J Basic Clin Physiol Pharmacol 2003; 14(2):95-118.

18. Perez R, Freeman S, Sohmer H. Effect of an initial noise induced hearing loss on subsequent noise induced hearing loss. Hear Res.2004; 192(1-2):101106.

19. Almadori G, Ottaviani F, Paludetti G, Rosignoli M, Gallucci L, D’Alatri L, Vergoni G. Auditory brainstem responses in noise-induced permanent hearing loss. Audiology 1988; 27(1):36-41.

20. Musiek FE, Borenstein SP, Hall III JW, Schwaber MK. Audiometria de tronco encefálico (ABR): neurodiagnóstico e aplicações intraoperatórias. In: Katz, J. Tratado de audiologia clínica. 4aed. São Paulo: Manole; 1999. p. 349-371.

21. Ferreira LLA, Silveira JAM, Percebo CC, Gonçalez F. Perda auditiva induzida por ruído: análise dos achados de audiometria tonal, potenciais evocados auditivos de tronco cerebral e emissões otoacústicas evocadas por produto de distorção. Rev Brasileira de Otorrinolaringologia 2001; 67(1):9-14.

Artigo apresentado em 26/03/2007

Aprovado em 23/11/2007

Versão final apresentada em 20/12/2007 